\title{
Case Report: Shewanella Algae Pneumonia and Bacteremia in an Elderly Male Living at a Long-Term Care Facility
}

\author{
Thomas J. Weiss, ${ }^{1 \star}$ Javier J. Barranco-Trabi, ${ }^{2}$ Aaron Brown, ${ }^{2}$ Tiffany T. Oommen, ${ }^{2}$ Victoria Mank, ${ }^{2}$ and Cameron Ryan ${ }^{2}$ \\ ${ }^{1}$ Uniformed Services University of the Health Sciences, Bethesda, Maryland; ${ }^{2}$ Department of Internal Medicine, Tripler Army Medical Center, \\ Honolulu, Hawaii
}

\begin{abstract}
Shewanella algae is a gram-negative, nonfermenting, oxidase-positive, motile bacillus that is ubiquitous in aquatic ecosystems. Human infections are rare and the immunocompromised are left most vulnerable. Risk factors for this infection include exposure to seawater, consumption of raw seafood, and underlying comorbid conditions such as hepatobiliary disease and chronic cutaneous ulcers. Previously documented cases of $S$. algae have involved near drownings, contaminated raw shellfish, or wound exposure to seawater, mud, sand, and sewage. This case study is unique in that it describes Shewanella bacteremia without any of these typical preceding exposures. We present a case of $S$. algae pneumonia and bacteremia in an elderly male patient living at a long-term care facility without any recent open water exposure.
\end{abstract}

\section{INTRODUCTION}

Shewanella algae are heterotrophic facultative anaerobes that are widely distributed in aquatic habitats (Figure 1). Typically, this infection is seen among the immunocompromised population in the setting of open water trauma. ${ }^{1,2}$ Cases of Shewanella have been reported worldwide but are more common in the tropics and during summer months in temperate zones where people are more often in contact with the water. ${ }^{3}$ Previously reported cases suggest that $S$. algae can

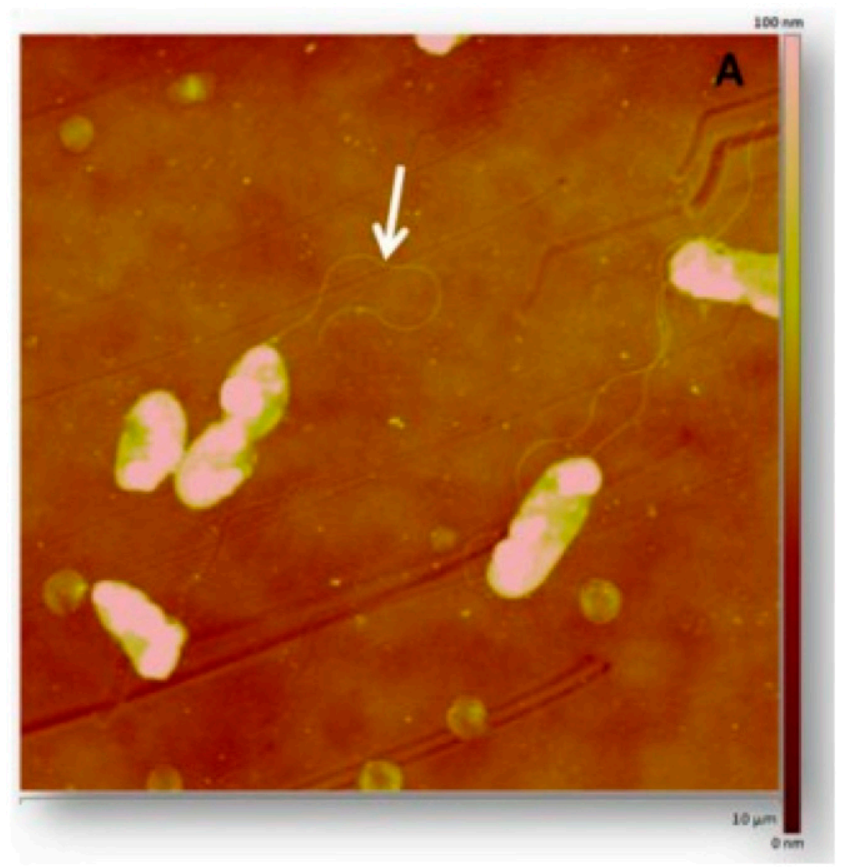

FIGURE 1. Shewanella algae morphology and identifiable singular flagella. ${ }^{5}$ This figure appears in color at www.ajtmh.org.

\footnotetext{
*Address correspondence to Thomas J. Weiss, Uniformed Services University of the Health Sciences (USUHS), 4301 Jones Bridge Rd., Bethesda, MD 20814. E-mail: thomas.weiss@usuhs. edu
}

cause otitis externa, soft tissue infections, bacteremia, and hepatobiliary infections. The organism is often susceptible to third generation cephalosporins, $\beta$-lactam $/ \beta$-lactamase inhibitors, aminoglycosides, and fluroquinolones. ${ }^{4}$ Specific comorbidities associated with Shewanella infections include chronic liver and kidney disease, severe peripheral vascular disease (PVD), and skin and soft tissue infections (SSTI). ${ }^{1}$

\section{CASE REPORT}

Patient is a 73-year-old Caucasian male, with past medical history significant for asthma, chronic obstructive pulmonary disease (COPD), allergic bronchopulmonary aspergillosis, coronary artery disease (CAD), diabetes mellitus type II, and recent acute cholecystitis complicated by sepsis, and treated with a percutaneous cholecystostomy drain. He is a resident of a long-term care facility who initially presented to the emergency department for acute onset of altered mental status, fever $\left(101.4^{\circ} \mathrm{F}\right)$, tachycardia (110 bpm), dyspnea, and hypoxia. Initial laboratory evaluation was notable for leukocytosis (19.4) with neutrophil predominance (89.2\%), elevated blood urea nitrogen (31), elevated glucose (228), elevated procalcitonin (0.51), and negative COVID-19 test. Chest X-ray demonstrated a left lower lobe pneumonia with associated pleural effusion and atelectasis (Figure 2). Patient was admitted into the hospital with suspected pneumosepsis and empiric antibiotic therapy was initiated with doxycycline and cefepime (to provide broad coverage for methicillinresistant Staphylococcus aureus, Pseudomonas and atypical organisms). Blood samples were drawn from the patient and both aerobic cultures resulted in the growth of S. algae. Antibiotic susceptibility was assessed via Kirby Bauer disc diffusion assay. The data is displayed in Table 1 . The patient improved clinically and the antibiotic regimen was then narrowed to ceftriaxone based on both infectious disease literature and laboratory antibiotic susceptibilities. After completion of the course of antimicrobials, the patient had resolution of all symptoms and was discharged from the hospital.

\section{DISCUSSION}

Shewanella algae infections are most common following traumatic injuries and near drownings in both freshwater and 


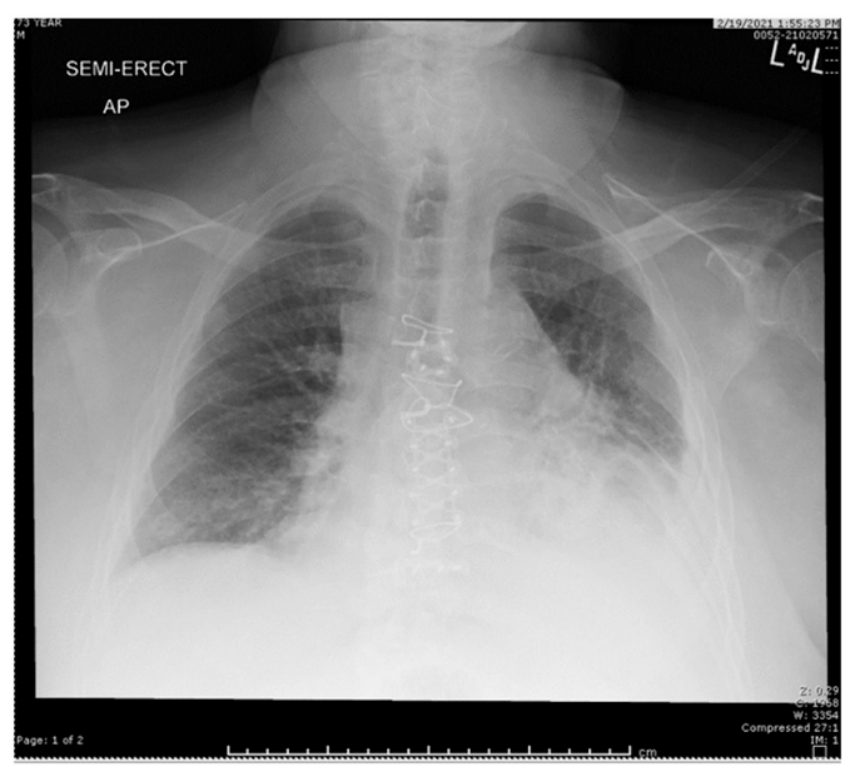

FIGURE 2. Chest X-ray obtained upon initial presentation. Notable for left lower lobar consolidation with associated pleural effusion, and atelectasis.

saltwater. ${ }^{6}$ Tourists, swimmers, fisherman, and sailors with underlying liver disease and immunocompromising conditions are at highest risk of contracting a water-related soft tissue or pulmonary Shewanella infection. ${ }^{7-9}$ This patient's exposure to $S$. algae is not easily identified. One potential avenue of infection would be aspiration of a contaminated water supply at the long-term care facility. However, there are no previous case studies describing incidents of aspiration pneumonia caused by S. algae. Another possible source of infection would be ingestion of raw seafood, though the patient was unable to recall recent ingestion of any such foods. ${ }^{10}$ Exposure of cutaneous ulcers to seawater is also a well-documented source of infection; however, this patient had no open skin ulcers or seawater exposure in the last 6 months. As such, clinicians should broaden their awareness of this organism's potential routes of inoculation, predisposing factors for infection, and the wide variety of clinical presentations associated with S. algae.

TABLE 1

Antibiotic sensitivity assessed via Kirby Bauer disc assay*

\begin{tabular}{lcc}
\hline \multicolumn{1}{c}{ Antibiotic used } & Zone of inhibition $(\mathrm{mm})$ & Sensitivity \\
\hline Ceftazidime & 29 & Sensitive \\
Ciprofloxacin & 27 & Sensitive \\
Gentamicin & 23 & Sensitive \\
Piperacillin/Tazobactam & 30 & Sensitive \\
Tobramycin & 21 & Sensitive \\
Imipenem & 18 & Sensitive \\
Amikacin & 20 & Sensitive \\
Ampicillin & 20 & Sensitive \\
Ampicillin/Sulbactam & 24 & Sensitive \\
Sulfamethoxazole/Trimethoprim & 24 & Sensitive \\
Cefazolin & 0 & Resistant \\
Ceftriaxone & 26 & Sensitive \\
\hline${ }^{*}$ MIC results were unable to be obtained. & &
\end{tabular}

\section{CONCLUSION}

This case presentation describes a unique situation in which a patient develops $S$. algae bacteremia without any identifiable preceding exposures. Though human infection remains rare, the incidence is increasing, and this case highlights the importance of considering Shewanella spp. as the cause of infection in settings where the patient may have been exposed to seawater or raw shellfish. This case also brings to light the question of whether potable water may serve as a source of infection and whether additional testing for contamination is indicated.

Received June 1, 2021. Accepted for publication August 18, 2021.

Published online November 15, 2021.

Acknowledgments: We would like to thank Dr. Mary Saleeby (Internal Medicine attending at Tripler Army Medical Center) for reviewing and advising on the preparation of the manuscript.

Authors' addresses: Thomas J. Weiss, Uniformed Services University of the Health Sciences, Bethesda, MD, E-mail: thomas.weiss@ usuhs.edu. Javier J. Barranco-Trabi, Aaron Brown, Tiffany T. Oommen, Victoria Mank, and Cameron Ryan, Department of Internal Medicine, Tripler Army Medical Center (TAMC), Honolulu, HI, E-mails: javier.barranco-trabi.civ@mail.mil, aaron.j.brown131.mil@ mail.mil, tiffany.t.oommen.mil@mail.mil, victoria.m.mank.mil@mail. mil, and cameron.m.ryan.mil@mail.mil.

This is an open-access article distributed under the terms of the Creative Commons Attribution (CC-BY) License, which permits unrestricted use, distribution, and reproduction in any medium, provided the original author and source are credited.

\section{REFERENCES}

1. Srinivas J, Pillai M, Vinod V, Dinesh RK, 2015. Skin and soft tissue infections due to Shewanella algae - an emerging pathogen. J Clin Diagn Res 9: DC16-DC20.

2. Holt HM, Gahrn-Hansen B, Bruun B, 2005. Shewanella algae and Shewanella putrefaciens: clinical and microbiological characteristics. Clin Microbiol Infect 11: 347-352.

3. Bernshteyn M, Ashok Kumar P, Joshi S, 2020. Shewanella algae - a novel organism causing bacteremia: a rare case and literature review. Cureus 12: e10676.

4. Vignier $\mathrm{N}$ et al., 2013. Human infection with Shewanella putrefaciens and S. algae: report of 16 cases in Martinique and review of the literature. Am J Trop Med Hyg 89: 151-156.

5. Martín-Rodríguez AJ, González-Orive A, Hernandez-Creus A, Morales A, Dorta-Guerra R, Norte M, Martin VS, Fernández $\mathrm{JJ}, 2014$. On the influence of the culture conditions in bacterial antifouling bioassays and biofilm properties: Shewanella algae, a case study. BMC Microbiol 14: 1-5.

6. Diaz JH, Lopez FA, 2015. Skin, soft tissue and systemic bacterial infections following aquatic injuries and exposures. Am J Med Sci 349: 269-275.

7. Baddour LM, 1992. Extraintestinal Aeromonas infections-looking for Mr. Sandbar. Mayo Clin Proc 67: 496-498.

8. Tacket CO, Brenner F, Blake PA, 1984. Clinical features and an epidemiological study of Vibrio vulnificus infections. J Infect Dis 149: 558-561.

9. Myung DS, Jung YS, Kang SJ, Song YA, Park KH, Jung SI, Kim $\mathrm{SH}$, Shin JH, 2009. Primary Shewanella algae bacteremia mimicking Vibrio septicemia. J Korean Med Sci 6: 1192-1194.

10. Tseng SY, Liu PY, Lee YH, Wu ZY, Huang CC, Cheng CC, Tung KC, 2018. The pathogenicity of Shewanella algae and ability to tolerate a wide range of temperatures and salinities. Can $J$ Infect Dis Med Microbiol 2018: 6976897. 